\title{
Sex Differences in COMT Polymorphism Effects on Prefrontal Inhibitory Control in Adolescence
}

\begin{abstract}
Thomas P White*,', Eva Loth ${ }^{2}$, Katya Rubia ${ }^{3}$, Lydia Krabbendam ${ }^{4}$, Robert Whelan ${ }^{5,6}$, Tobias Banaschewski ${ }^{7}$, Gareth J Barker $^{8}$, Arun LW Bokde ${ }^{9}$, Christian Büchel ${ }^{10}$, Patricia Conrod ${ }^{8,11}$, Mira Fauth-Bühler ${ }^{7,12}$, Herta Flor ${ }^{7}$, Vincent Frouin ${ }^{13}$, Jürgen Gallinat ${ }^{14}$, Hugh Garavan ${ }^{5,6}$, Penny Gowland ${ }^{15}$, Andreas Heinz ${ }^{14}$, Bernd Ittermann ${ }^{16}$, Claire Lawrence ${ }^{17}$, Karl Mann ${ }^{7}$, Marie-Laure Paillère ${ }^{18,19}$, Frauke Nees ${ }^{5}$, Tomas Paus ${ }^{17,20,21}$, Zdenka Pausova ${ }^{22}$, Marcella Rietschel ${ }^{7}$, Trevor Robbins ${ }^{23}$, Michael N Smolka ${ }^{24}$, Sukhwinder S Shergill ${ }^{1,25}$, Gunter Schumann ${ }^{2,25}$ and the IMAGEN Consortium

'Department of Psychosis Studies, Institute of Psychiatry, King's College London, London, UK; ${ }^{2}$ MRC Social, Genetic and Developmental Psychiatry Centre, Institute of Psychiatry, King's College London, London, UK; ${ }^{3}$ Department of Child and Adolescent Psychiatry, Institute of Psychiatry, King's College London, London, UK; ${ }^{4}$ Department of Educational Neuroscience, Faculty of Psychology and Education, VU University Amsterdam, Amsterdam, The Netherlands; ${ }^{5}$ Institute of Neuroscience, Trinity College Dublin, Dublin, Ireland; ' $D$ Departments of Psychiatry and Psychology, University of Vermont, Burlington, VT, USA; ${ }^{7}$ Department of Cognitive and Clinical Neuroscience, Central Institute of Mental Health, Medical Faculty Mannheim, Heidelberg University, Mannheim, Germany; ${ }^{8}$ Institute of Psychiatry, King's College London, London, UK; ${ }^{9}$ Institute of Neuroscience and Discipline of Psychiatry, School of Medicine, Trinity College Dublin, Dublin, Ireland; ${ }^{10}$ Universitaetsklinikum Hamburg Eppendorf, Hamburg, Germany; "Department of Psychiatry, Universite de Montreal, CHU Ste Justine Hospital, Montreal, QC, Canada; ${ }^{12}$ Department of Addictive Behaviour and Addiction Medicine, Heidelberg University, Heidelberg, Germany; ${ }^{13}$ Neurospin, Commissariat à I'Energie Atomique et aux Energies Alternatives, Paris, France; ${ }^{14}$ Department of Psychiatry and Psychotherapy, Campus Charité Mitte, CharitéUniversitätsmedizin Berlin, Berlin, Germany; ${ }^{15}$ Department of Physics and Astronomy, University of Nottingham, Nottingham, UK; ${ }^{16}$ PhysikalischTechnische Bundesanstalt (PTB), Braunschweig und Berlin, Berlin, Germany; ${ }^{17}$ School of Psychology, University of Nottingham, Nottingham, UK; ${ }^{18}$ Institut National de la Santé et de la Recherche Médicale, INSERM CEA Unit 1000 'Imaging \& Psychiatry', University Paris Sud, Orsay, France; ${ }^{19}$ AP-HP Department of Adolescent Psychopathology and Medicine, Maison de Solenn, University Paris Descartes, Paris, France; ${ }^{20}$ Rotman Research Institute, University of Toronto, Toronto, ON, Canada; ${ }^{21}$ Montreal Neurological Institute, McGill University, Montreal, QC, Canada; ${ }^{22}$ The Hospital for Sick Children, University of Toronto, Toronto, ON, Canada; ${ }^{23}$ Behavioural and Clinical Neurosciences Institute, Department of Experimental Psychology, University of Cambridge, Cambridge, UK; ${ }^{24}$ Department of Psychiatry and Neuroimaging Center, Technische Universität Dresden, Dresden, Germany
\end{abstract}

Catecholamine-0-methyl-transferase (COMT) gene variation effects on prefrontal blood oxygenation-level-dependent (BOLD) activation are robust; however, despite observations that COMT is estrogenically catabolized, sex differences in its prefrontal repercussions remain unclear. Here, in a large sample of healthy adolescents stratified by sex and Val ${ }^{158}$ Met genotype $(n=|| 33)$, we examine BOLD responses during performance of the stop-signal task in right-hemispheric prefrontal regions fundamental to inhibitory control. A significant sex-by-genotype interaction was observed in pre-SMA during successful-inhibition trials and in both pre-SMA and inferior frontal cortex during failed-inhibition trials with Val homozygotes displaying elevated activation compared with other genotypes in males but not in females. BOLD activation in the same regions significantly mediated the relationship between COMT genotype and inhibitory proficiency as indexed by stop-signal reaction time in males alone. These sexually dimorphic effects of COMT on inhibitory brain activation have important implications for our understanding of the contrasting patterns of prefrontally governed psychopathology observed in males and females.

Neuropsychopharmacology (20I4) 39, 2560-2569; doi:I0.1038/npp.20I4.I07; published online I8 June 2014

*Correspondence: Professor G Schumann, MRC Social, Genetic and Developmental Psychiatry Centre, Institute of Psychiatry, de Crespigny Park, London SE5 8AF, UK, Tel: +I 22644207848 5314, Fax: + I 22644207848 0940, E-mail: gunter.schumann@kcl.ac.uk ${ }^{25}$ These authors contributed equally to this work.

Received II September 2013; revised 3 April 2014; accepted 4 April 20।4; accepted article preview online I 3 May 2014

\section{INTRODUCTION}

Inhibitory control indispensably underlies executive function. Sex differences are evident in its proficiency: men score higher for traits of impulsivity than women (Campbell and Muncer, 2009; Labouvie and McGee, 1986); and are more likely to experience disorders of impulse control, such 
as attention deficit hyperactivity disorder (ADHD), substance abuse, and conduct disorder (Eme, 2007; Kessler et al, 2007; Newman et al, 2005). Fronto-striatal dopamine dysregulation is recognized as central to these disorders (Hart et al, 2013; Rubia et al, 2011). Furthermore, prefrontal dopaminergic function is intimately affected by genetic variation in dopamine transporting and metabolizing enzymes, the most prominent being catecholamine- 0 methyl-transferase (COMT). Nevertheless, the precise nature of the relationship between sex, COMT variation, and inhibitory control remains unclear.

Converging lines of molecular, behavioral and neuroimaging evidence demonstrate the functional effects of COMT-gene variation. COMT regulates brain function via synaptic catabolism of catecholamine neurotransmitters including dopamine and noradrenaline. The COMT gene is found on chromosome 22q11; its membrane-bound isoform (mb-COMT) has a high affinity for dopamine and is influential prefrontally on account of a local shortage of dopamine transporter protein (Yavich et al, 2007).

$\mathrm{Val}^{158} \mathrm{Met}-\mathrm{a}$ G/A substitution in mb-COMT at codon 158 producing either the ancestral valine or methioninesubstantially affects COMT activity and is the most studied COMT functional polymorphism (Tunbridge and Harrison, 2010). The Val form is more stable and, thus, more active than Met at body temperature; Met homozygotes exhibit a $40 \%$ reduction in COMT activity in prefrontal cortex as compared with Val-Val individuals (Chen et al, 2004). Evidence suggests that this difference shapes cognitive performance. Met has been somewhat inconsistently associated with improved prefrontally controlled working memory, attention, and executive function (Barnett et al, 2007; Egan et al, 2001; Mier et al, 2010; Wardle et al, 2013). More robust are demonstrations from blood oxygenationlevel-dependent (BOLD) data of increased prefrontal activation (often interpreted in terms of reduced efficiency) in individuals with the Val allele during cognitive processing (Mier et al, 2010). Alternatively, Met has been ascribed advantage in the tonic stimulation of cortical D1 receptors leading to improved stabilization of relevant information; by contrast, Val has been postulated to tune phasic stimulation of striatal D2 receptors, enhancing the rapid updating crucial to processes such as response inhibition (Bilder et al, 2004). This elegant model currently receives little empirical support. To the contrary, behavioral examination has revealed Met advantages during stable conditions but no behavioral COMT effects in conditions requiring rapid updating (Rosa et al, 2010). Nevertheless, as this study did not employ cerebral recordings, further investigation is warranted.

Sex appears to moderate COMT effects on cognition (Harrison and Tunbridge, 2008). In a behavioral study of 8-10 year olds $(n=5000)$, Met was associated with better function in several domains including attention and verbal intelligence, with these effects greater in or limited to boys (Barnett et al, 2007). The same group extended their study of COMT polymorphism effects in the same data to confirm this sex-by-genotype interaction on verbal intelligence quotient (IQ) (three-SNP haplotype), and observed a sexby-genotype interaction on working memory (rs165599) (Barnett et al, 2009). Furthermore, anatomical support for these effects is provided by sex-by-genotype interactions on dorsal and orbital frontal volumes in adolescents with the microdeletion-induced velo-cardio-facial syndrome (who are, therefore, Met or Val hemizygous), whereby Met carriers exhibit greater volumes if male but smaller volumes if female (Kates et al, 2006). Sex-by-genotype interactions on prefrontal BOLD activation have also been observed in the same disorder, whereby male Met carriers exhibit elevated activation as compared with male Val carriers in right pars opercularis during the processing of pleasant stimuli, but female Val carriers display enhanced activation as compared with female Met carriers (Coman et al, 2010).

In healthy individuals, sex differences in COMT effects on amygdalar BOLD reactivity during emotional processing (Domschke et al, 2012) and widespread morphometric features (Zinkstok et al, 2006) have also been previously observed; however, to our knowledge, the influence of sex on COMT effects on normative prefrontal BOLD responses has not been directly investigated. Here, we examine sex moderation of prefrontal indices of inhibitory control during performance of the stop-signal task (SST) in a large group of healthy adolescents split according to sex and $\mathrm{Val}^{158}$ Met genotype. Performance of the SST depends on rapid updating and inhibition of goal-driven cerebral activity, as the participant is unpredictably required to completely suppress a motor response that has already been triggered and is in the process of being executed. Investigation of adolescent individuals is undertaken with the aim of establishing the physiological foundations of gender differences in the progressive maturation of inhibitory networks with age (Rubia et al, 2013). We focus on two regions previously implicated as fundamental cortical substrates of response inhibition: right-hemispheric inferior frontal cortex (IFC) and pre-SMA (Aron and Poldrack, 2006). The extant literature suggests that during inhibition the pre-SMA generates the control signal and that, by contrast, the right IFC implements inhibitory control (Aron, 2011; Aron and Poldrack, 2006).

Robust evidence from meta-analysis of activation in diverse cognitive settings (Mier et al, 2010) drove our prediction that Met carriers would exhibit lower prefrontal BOLD activation compared with Val carriers. Given sex differences in circulating estrogen levels and the catabolic effects of estrogen on COMT (Cohn and Axelrod, 1971; Xie et al, 1999), we predicted that COMT effects would be larger in boys. Sex modulation of behavioral COMT effects is more difficult to foretell on account of the inconsistent human literature (Barnett et al, 2009; Wardle et al, 2013); nevertheless, we tentatively predicted similar sexually dimorphic COMT effects on indices of inhibitory performance. Our final hypothesis-made with the aim of elucidating the behavioral implications of putative physiological differences-was that that BOLD activity during inhibition would be a more consistent mediating mechanism of the relationship between COMT and inhibitory performance in boys than in girls.

\section{MATERIALS AND METHODS}

This work was conducted as part of the multicentre IMAGEN project, whose standard operating procedures in terms of ethics, recruitment, consent, test details, blood 
sampling and storage, and genotyping are available elsewhere (Schumann et al, 2010).

\section{Participants}

In total, 1133 adolescents from the IMAGEN sample were included in this study. Details of individuals recruited for the IMAGEN study but excluded from this work are presented in Supplementary Table S1. Sex/COMT-genotype distributions of included individuals are presented by study site in Table 1(A). The $\mathrm{Val}^{158}$ Met genotype distribution did not differ significantly from the expected numbers calculated on the basis of observed allele frequencies according to the Hardy-Weinberg equilibrium $\left(\chi^{2}<0.01, P>0.99\right)$. All analyses presented in this report include data from all 1133 individuals unless otherwise explicitly stated.

Age-normalized IQ was determined by administration of selected subscales of the Wechsler intelligence battery for children (Wechsler et al, 1992). Verbal IQ was estimated via the vocabulary and similarities subscales; performance IQ was evaluated using the block-design and matrix-reasoning subscales. Tanner staging of physical maturity (Tanner, 1962) was assessed with the sex-specific, self-rated Pubertal Developmental Scale questionnaire (Carskadon and Acebo, 1993). In addition, in light of their relevance to inhibitory function, ADHD traits (hyperactivity, inattention, and impulsivity) were assessed in this non-clinical sample using parent-ratings on the Strengths and Difficulties Questionnaire (SDQ; http://www.sdqinfo.com). Demographic data are presented in Table 1(B). General linear models (GLMs) to assess the effects of sex and genotype on discrete demographic and clinical traits demonstrated that age, parental ethnicity, performance IQ, and ADHD-related characteristics did not significantly differ on the basis of sex or genotype. The sample's ADHD-traits score of $2.84 \pm$ 2.25 (mean \pm SD) demonstrates the current participants' good health in this domain when considered in comparison with a recent European multicentre study of parent-rated

Table I Sample Demography and Task Performance

(A) Sex-genotype frequencies by center

\begin{tabular}{|c|c|c|c|c|c|c|c|c|c|}
\hline Genotype & $\begin{array}{c}\text { Site I } \\
\text { M/F }\end{array}$ & $\begin{array}{c}\text { Site } 2 \\
\text { M/F }\end{array}$ & $\begin{array}{c}\text { Site } 3 \\
\text { M/F }\end{array}$ & $\begin{array}{c}\text { Site } 4 \\
\text { M/F }\end{array}$ & $\begin{array}{c}\text { Site } 5 \\
\text { M/F }\end{array}$ & $\begin{array}{c}\text { Site } 6 \\
\text { M/F }\end{array}$ & $\begin{array}{c}\text { Site } 7 \\
\text { M/F }\end{array}$ & $\begin{array}{c}\text { Site } 8 \\
\text { M/F }\end{array}$ & $\begin{array}{c}\text { Total } \\
\text { M/F }\end{array}$ \\
\hline Val-Val & $25 / 18$ & 19/25 & $10 / 10$ & I5/20 & $12 / 12$ & $17 / 19$ & $23 / 14$ & $8 / 19$ & $129 / 135$ \\
\hline Val-Met & $34 / 37$ & $48 / 47$ & $27 / 22$ & $39 / 36$ & $42 / 37$ & $30 / 26$ & $31 / 36$ & $37 / 37$ & $288 / 278$ \\
\hline Met-Met & $17 / 16$ & $21 / 22$ & $7 / 11$ & $16 / 19$ & 28/29 & $19 / 14$ & $12 / 20$ & $21 / 3 \mid$ & $141 / 162$ \\
\hline
\end{tabular}

(B) Demographic and neuropsychological characteristics

\begin{tabular}{|c|c|c|}
\hline \multirow[t]{2}{*}{ Measure } & \multicolumn{2}{|c|}{ Sex } \\
\hline & Male & Female \\
\hline Handedness (left/right) & $65 / 482$ & $59 / 508$ \\
\hline Mother's ethnicity (white/other) & $550 / 8$ & $569 / 6$ \\
\hline Performance IQ & $108.12(15.09)$ & $107.49(14.03)$ \\
\hline Pubertal development scale & $3.17(0.78)$ & $3.99(0.43)$ \\
\hline ADHD traits (SDQ) & $3.22(2.33)$ & $2.47(2.11)$ \\
\hline
\end{tabular}

(C) Task performance by sex and genotype

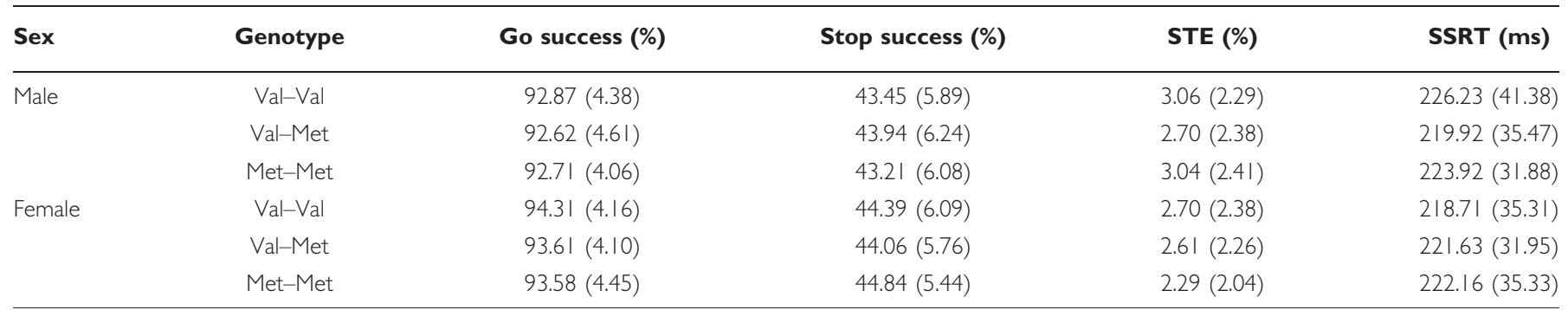

Abbreviations: ADHD, attention deficit hyperactivity disorder; IQ, intelligence quotient; SDQ, Strengths and Difficulties Questionnaire (http://www.sdqinfo.com); SSRT, stop-signal reaction time; STE, stop too early.

Mean values with SDs are bracketed. 
SDQ scores in ADHD (control group aged 5-15 years $(n=10298): 3.5 \pm 2.6$; ADHD group aged $11-18$ years $(n=334) 8.1 \pm 1.8)$ (Becker et al, 2006). In the current sample, sex significantly predicted verbal IQ (likelihoodratio $\chi^{2}=8.71, P=0.003$ ) and pubertal stage (likelihoodratio $\left.\chi^{2}=349.43, P<1 \times 10^{-5}\right)$. Post hoc $t$-tests revealed that boys exhibited higher verbal IQ compared with girls $(\mathrm{T}(1087)=2.652, P=0.008)$, and that girls were significantly more developed than boys $(\mathrm{T}(856.01)=21.95$, $\left.P<1 \times 10^{-5}\right) . \quad \chi^{2}$ testing established that handedness, established by participant self-report, did not significantly differ by sex or genotype.

\section{SST}

The SST was adapted from Rubia et al (2003, 2007). Participants were instructed to make a single, button-press response to either of two regularly presented, visual-go stimuli (arrows pointing left or right shown centrally for $1000 \mathrm{~ms}$ ), unless followed by an unpredictable stop signal (arrow pointing upwards shown for 100-300 ms). A tracking algorithm (Rubia et al, 2003, 2007) moderated task difficulty by varying the stop-signal delay, which was $250-900 \mathrm{~ms}$ (in 50 -ms increments), on the basis of participant performance. The paradigm contained 400 go trials interspersed with 80 stop trials; between three and seven go trials occurred between stop trials. The inter-trial interval was jittered between 1.6 and $2.0 \mathrm{~s}$ (mean: $1.8 \mathrm{~s}$ ) to enhance statistical efficiency (Dale, 1999). If the participant responded to the go-stimulus before stop-stimulus presentation (stop too early; STE), then the trial was repeated for up to a maximum of seven trials. GLMs were used to assess between-subject effects of sex and genotype on go-success rate, stop-success rate, and STE rate, while accounting for performance IQ and paternal ethnicity (see Supplementary Information 1 for covariate justification).

\section{Calculation of Stop-Signal Reaction Time (SSRT)}

SSRT is the time taken to cancel a movement after stopstimulus presentation. As such, a low SSRT represents good inhibitory function. SSRT was calculated by subtracting the mean stop-signal delay from the Go RT at the percentile corresponding to the proportion of unsuccessfully inhibited stop trials (as per previous analysis of this dataset (Whelan et $a l, 2012)$ ). This was done under the rationale of the horse-race model of inhibition (Logan et al, 1984), whereby successful inhibition signifies the stop process being completed before the go response.

Repetition of stop trials on account of STE responses can affect the accuracy of SSRT calculation because STE frequency varied between participants. Furthermore, as trial repetition was constrained to a maximum of seven, the number of valid stop trials also varied. For participants with more than eight STEs, SSRT was calculated up to the eighth STE. Participants producing eight STEs prior to the 300th trial were excluded from SSRT calculation. In total, SSRT was calculated for 630 participants. Consequently, all analyses involving SSRT include 630 individuals. GLM-testing assessed between-subject effects of sex and genotype on SSRT, and the linear effects of ADHD-related problems on SSRT, accounting for no covariates. (Supplementary
Information 2 investigates demographic and task-performance differences between those individuals included in and those excluded from the SSRT-focused analyses.)

\section{fMRI Acquisition and Analysis}

The MR scanning protocols, their cross-site standardization and quality checks, and preprocessing of resulting data are described elsewhere (Schumann et al, 2010; Whelan et al, 2012). fMRI data were analyzed using SPM8 (Statistical Parametric Mapping, http://www.fil.ion.ucl.ac.uk/spm/). First-level activation maps were computed for stop-success trials ( $v s$ baseline) and stop-fail trials ( $v s$ baseline) separately using individually specified GLMs. These contrasts were selected for analysis with the aim of characterizing independently the cerebral milieu associated with these conditions rather than differential activation of associated processes. (Additional contrasts are investigated in Supplementary Information 3.) Design matrices included regressors for go-success trials, stop-success trials, stop-fail trials, trials on which the go response was too late, trials on which the go response was wrong (if any), and realignment parameters as nuisance regressors. STE trials were included as go-success trials within the model. Task regressors were convolved with a canonical hemodynamic response function.

Region-of-interest (ROI) analyses were conducted on stop-success and stop-fail contrasts in IFC and presupplementary motor area (pre-SMA) of the right hemisphere. These regions are known to be the key cortical structures for inhibitory function required during the SST (Aron, 2011; Aron and Poldrack, 2006; Chambers et al, 2009). ROIs were generated with the WFU Pickatlas (Maldjian et al, 2003) and defined using automated anatomical labels (Tzourio-Mazoyer et al, 2002) as per Aron and Poldrack, 2006: IFC comprised the pars triangularis and pars opercularis compartments; pre-SMA consisted of the SMA compartment $(y>0)$. Mean contrast values within these ROIs were $\mathrm{Z}$-transformed by center prior to between-subject inference testing (Tahmasebi et al, 2012) to minimize potential between center differences in the magnitude of MRI effects.

GLMs were used to examine between-subject effects of $\mathrm{Val}^{158}$ Met genotype, sex, and their interaction on cortical efficiency for both contrasts and both ROIs independently, while accounting for the effects of performance IQ (as per Supplementary Information 1). Significance was ascribed according to a Bonferroni-corrected $\alpha$ of 0.0125 . This value was corrected for the number of contrasts and ROIs tested. Results nominally significant at conventional thresholds are also reported for illustration.

A series of exploratory whole-brain analyses of related phenomena were undertaken to complement these hypothesis-focused ROI analyses. (Their procedures are presented in Supplementary Information 4.)

\section{Moderated Mediation of Inhibitory Function}

To examine concurrently: (i) mediation by BOLD of COMT effects on inhibitory performance (indexed by individualspecific SSRT); and (ii) sex moderation of these effects, a moderated-mediation approach termed conditional process 


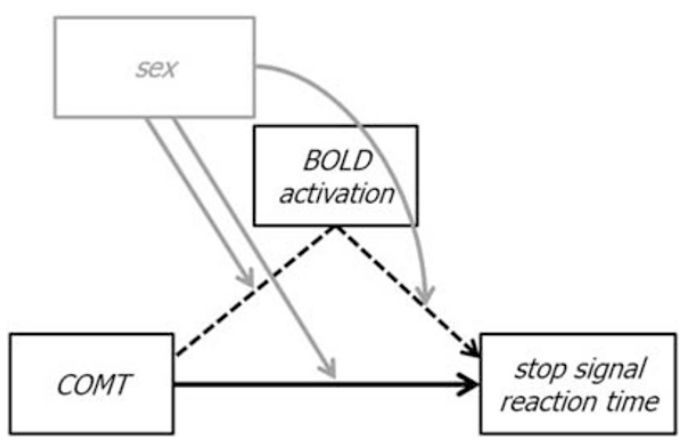

Figure I Conditional process analysis conceptual model. The horizontal line from COMT to hyperactivity represents the direct pathway; by contrast, the indirect pathway is indicated by the dotted line via BOLD activation. Conditional effects of sex were assessed on the pathways shown in grey.

analysis (CPA) was adopted using the PROCESS macro expansion (Hayes, 2013) for SPSS (SPSS, USA). CPA utilizes ordinary-least squares regression and permits the flexible evaluation of diverse statistical interdependencies. Figure 1 depicts the conceptualized CPA model examined. Further background, justification of model selection and results are presented in Supplementary Information 5 and 6. Effects were independently examined for pre-SMA and IFC during both successful and failed inhibition, while controlling for the effects of age, handedness, and IQ in each model.

Using the CPA approach, hypothesis testing of direct effects is calculated on the basis of the regression coefficient and its standard error. The significance of indirect effects is adjudged using percentile-based bootstrap confidence intervals. In total, 5000 bootstrap samples were collected by randomly resampling the whole-sample dataset. A bootstrapped estimate of the standard error of the indirect effect, defined as the SD of the 5000 bootstrapped coefficient estimates, was then calculated. To reduce effects relating to potential between-group inhomogeneities of variance, heteroscedasticity-consistent standard errors were calculated. Finally, lower and upper limits of 95\%-percentilebased confidence intervals were calculated; significant effects were inferred where this range did not cross zero.

This approach also modeled the effects (in terms of regression coefficients) of sex, COMT contrast- and ROIspecific BOLD activation, and interactions of these factors on SSRT. Again, significance was ascribed according to a Bonferroni-corrected $\alpha$ of 0.0125 , but nominally significant findings are also reported.

\section{RESULTS}

\section{Behavioral Performance}

Table 1 (C) presents behavioral performance indices. Significant sex effects were observed for go-success (likelihood-ratio $\chi^{2}=15.78, \quad P=7 \times 10^{-5}, \quad R=0.12$ ), stopsuccess (likelihood-ratio $\chi^{2}=5.65, P=0.017, R=0.07$ ) and STE rates (likelihood-ratio $\chi^{2}=12.30, \quad P=4 \times 10^{-4}$, $R=0.10)$. Post hoc $t$-tests confirmed that girls attained greater go-success $\left(\mathrm{T}(1132)=4.17, P=3 \times 10^{-5}, R=0.12\right)$ and stop-success $(\mathrm{T}(1132)=2.03, P=0.042, R=0.06)$ rates

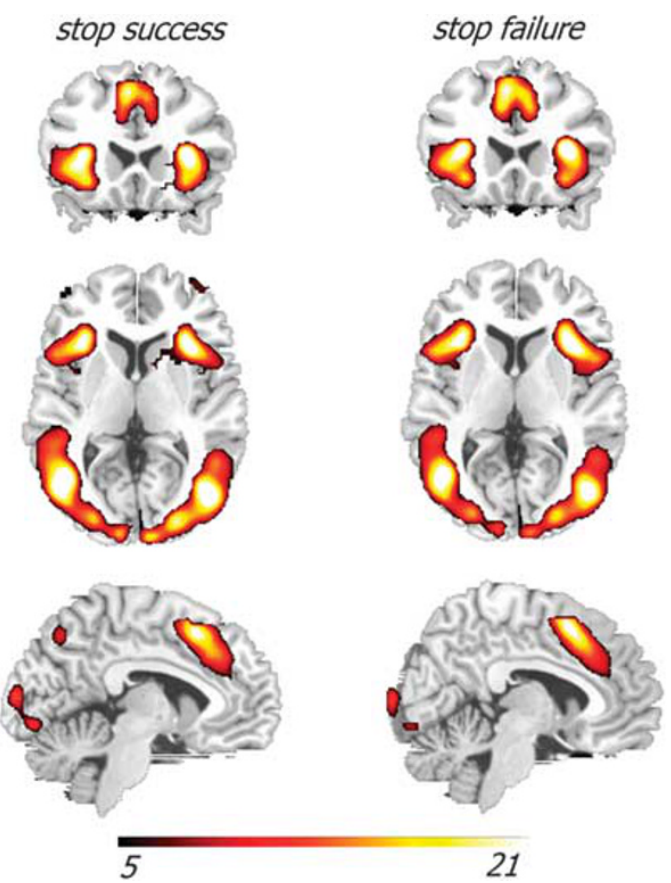

Figure 2 Regions significantly activated during stop-success (left) and stop-fail (right) trials overlaid on a standardized TI-weighted image. Color bar denotes $T$-scale. Voxels are thresholded at a family-wise error corrected $P$-value of 0.05

than boys. STE rate was, however, greater in boys than in girls $(\mathrm{T}(1132)=2.91, P=0.004, R=0.09)$. COMT genotype did not significantly predict these performance measures. SSRT did not significantly differ on the basis of sex, genotype, or the interaction of these factors.

\section{fMRI Results}

Figure 2 depicts regions activated during successful and failed inhibition (calculated as described in Supplementary Information 3). Significant gray matter foci are presented in Supplementary Tables S8-S11. Regions including bilateral IFC, pre-SMA, anterior insula and visual association cortices were significantly activated during both stop-success and stop-fail trials.

\section{Successful Inhibition}

Significant main effects of sex were observed in both preSMA (likelihood-ratio $\chi^{2}=12.34, P=4 \times 10^{-4}, R=0.10$ ) and IFC (likelihood-ratio $\chi^{2}=15.95, \quad P=7 \times 10^{-5}$, $\mathrm{R}=0.12$ ) during successful inhibition. Post hoc $t$-tests revealed responses in girls were smaller than those in boys (pre-SMA stop success: $\mathrm{T}(1132)=3.44, P=0.001, R=0.10$; IFC stop success: $\left.\mathrm{T}(1132)=4.15, P=4 \times 10^{-5}, R=0.12\right)$. No significant main effect of genotype was observed in either region during successful inhibition. However, a significant sex-by-genotype interaction (likelihood-ratio $\left.\chi^{2}=11.63, P=0.003, R=0.10\right)$ was observed during successful inhibition in the pre-SMA. In line with predictions, male Val homozygotes exhibited significantly greater 

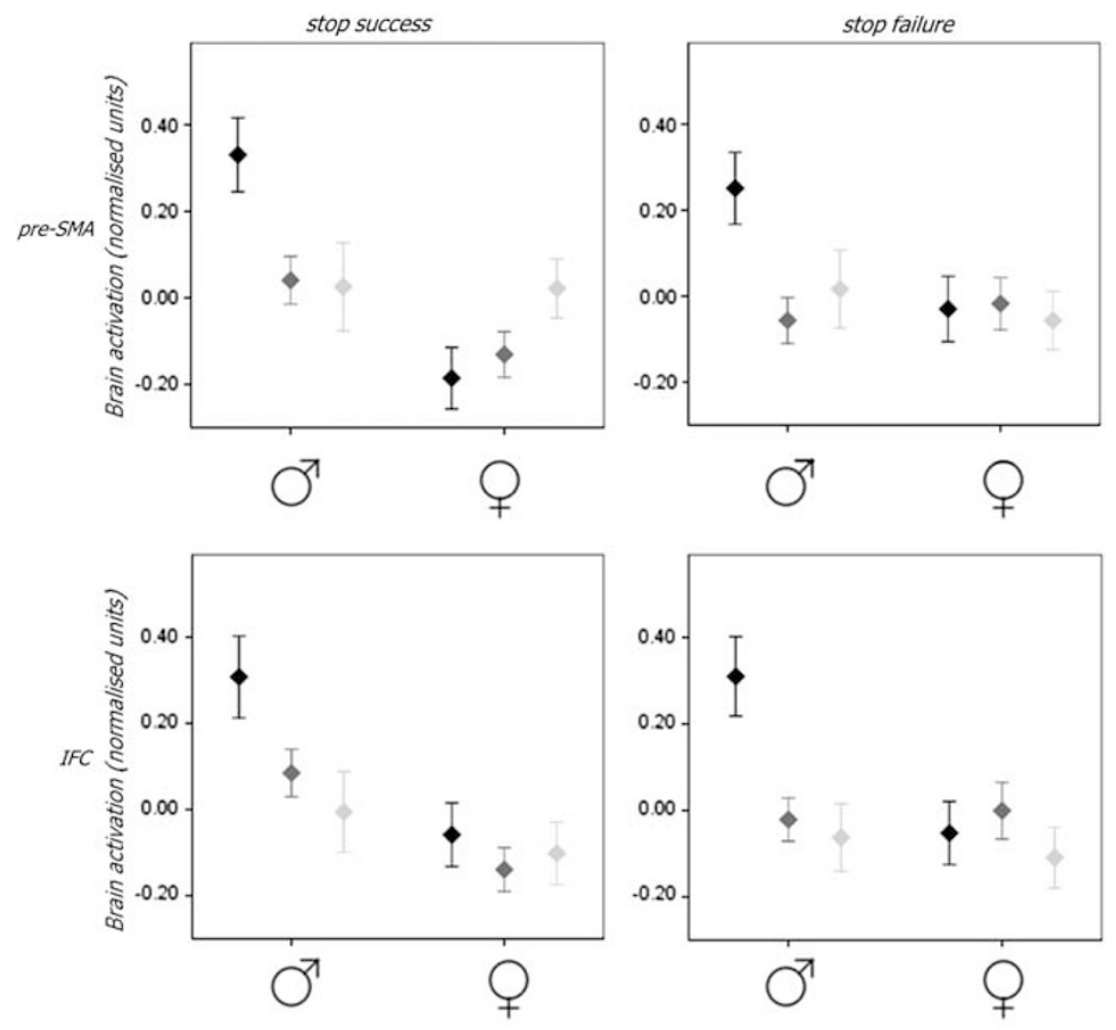

Val-Val Val-Met Met-Met

Figure 3 Group-averaged activation in pre-supplementary motor area (pre-SMA) and inferior frontal cortex (IFC) during stop trials for sex-genotype groups. Error bars denote SEM.

activity than male heterozygotes $(\mathrm{T}(416)=3.06, P=0.002$, $R=0.15)$ and male Met homozygotes $(\mathrm{T}(269)=2.64$, $P=0.009, R=0.16$ ); by contrast, there was some evidence of greater activation in pre-SMA in female Met homozygotes compared with female Val homozygotes $(\mathrm{T}(296)=$ 2.23, $\quad P=0.026, \quad R=0.13)$ and female heterozygotes $(\mathrm{T}(439)=1.71, \quad P=0.087, \quad R=0.08)$. These results are displayed in Figure 3.

\section{Failed Inhibition}

Nominally significant sex-by-genotype interactions were observed in IFC (likelihood-ratio $\chi^{2}=7.20, P=0.027$, $R=0.08$ ); and in pre-SMA (likelihood-ratio $\chi^{2}=7.72$, $P=0.021, R=0.08)$. These effects are accountable to significant differences between $\mathrm{Val}$ homozygotes and other participants in boys (IFC: Val-Val/Val-Met: $\mathrm{T}(416)=3.61, \quad P=3 \times 10^{-4}, \quad R=0.17 ; \quad$ Val-Val/Met-Met: $\mathrm{T}(268)=2.98, \quad P=0.003, \quad R=0.18 ; \quad$ pre-SMA: Val-Val/ Val-Met: $\mathrm{T}(416)=3.28, P=0.001, R=0.17$; Val-Val/MetMet: $\mathrm{T}(268)=2.10, P=0.037, R=0.18$ ), but non-significant between-group differences in females. Figure 3 illustrates these findings.

There was a nominally significant effect of sex in IFC (but not pre-SMA) during failed inhibition (likelihood-ratio $\left.\chi^{2}=5.79, \quad P=0.016, \quad R=0.07\right)$, with boys exhibiting increased activation compared with girls $(\mathrm{T}(1132)=1.90$, $P=0.059, R=0.06)$. Furthermore, a nominally significant effect of COMT genotype was observed on IFC (but not pre-SMA) activity during failed inhibition (likelihoodratio $\chi^{2}=7.38, P=0.025, R=0.08$ ), with greater activity in the Val homozygotes compared with heterozygotes $(\mathrm{T}(839)=2.52, P=0.012, R=0.09)$ and Met homozygotes $(\mathrm{T}(576)=2.58, P=0.010, R=0.11)$.

\section{Factors Modulating the Relationship Between SSRT and BOLD}

SSRT was neither significantly predicted by BOLD in pre-SMA nor IFC at a Bonferroni-corrected threshold. However, IFC activation during successful inhibition inversely predicted SSRT at an uncorrected threshold $(\beta=-9.96 \pm$ $4.65, \mathrm{~T}(629)=-2.14, P=0.033)$. Neither sex, COMT, nor their interaction predicted SSRT as assessed in any of the conducted CPA models. An exploratory whole-brain investigation of the relationship between SSRT and BOLD activation is presented in Supplementary Information 4.

The moderated-mediation analysis results are presented in Table 2. Whereas no significant direct effects of COMT were observed on inhibitory function as indexed by SSRT for either sex, consistent significant indirect effects of COMT on SSRT (via BOLD activation) were observed in boys for both IFC and pre-SMA during successful and failed inhibition. These effects were not significant in girls. 
Table 2 Conditional Direct and Indirect Effects of COMT-gene Variation on BOLD During Inhibition

(A) Conditional direct effects of COMT gene on hyperactivity

\begin{tabular}{|c|c|c|c|c|c|c|}
\hline ROI Model & Condition & Sex & Coefficient & SE & $\mathbf{T}$ & $P$ \\
\hline \multirow[t]{3}{*}{ pre-SMA } & Stop success & Boys & -1.66 & 3.25 & $-0.5 \mid$ & 0.609 \\
\hline & & Girls & 1.71 & 2.67 & -0.64 & 0.522 \\
\hline & & Girls & 1.53 & 2.68 & 0.57 & 0.568 \\
\hline \multirow[t]{2}{*}{ IFC } & Stop success & Boys & -1.72 & 3.27 & -0.53 & 0.599 \\
\hline & & Girls & 1.79 & 2.70 & 0.66 & 0.507 \\
\hline
\end{tabular}

(B) Conditional indirect effects of COMT gene on hyperactivity

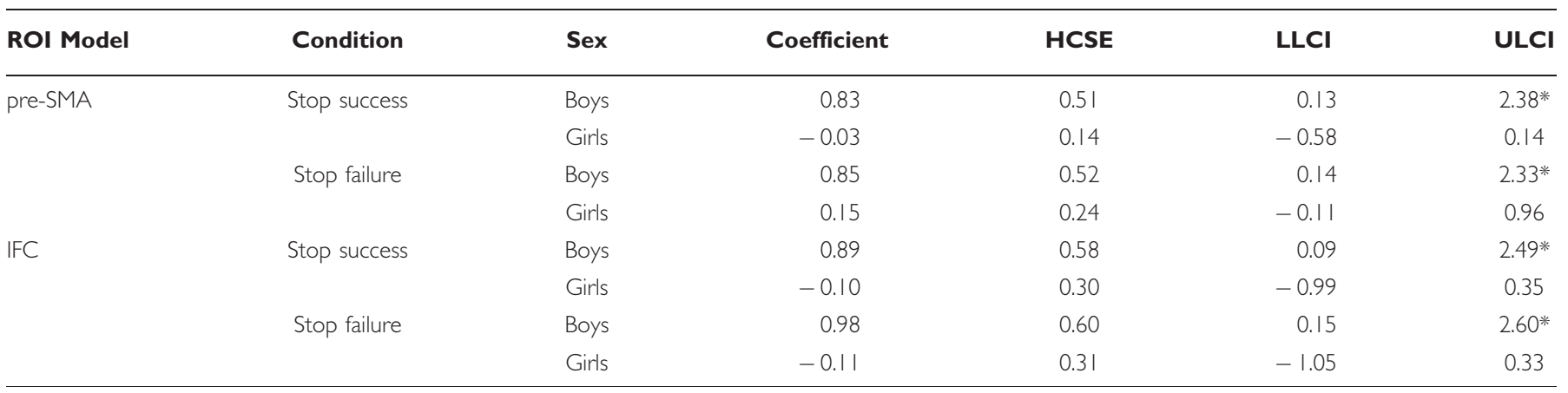

Abbreviations: HCSE, heteroscedasticity-consistent standard error, LLCl, lower-limit confidence interval; ROI, region-of-interest; SE, standard error; ULCl, upper-limit confidence interval.

Asterisks denote effects significant at an $\alpha$ of 0.05 .

\section{DISCUSSION}

The principal finding of this work is that sex significantly moderates COMT effects in the pre-SMA during successful inhibition; this is, therefore, the first normative study to demonstrate directly sex modulation of COMT effects on prefrontal activity. Male Val homozygotes exhibit significantly elevated activation compared with other male genotypes. By contrast, corresponding COMT effects are less robust in females. Similar sexual dimorphism is also seen in both right IFC and pre-SMA during failed inhibition. It is tempting to suggest that these findings empirically support the inverted U-shaped model of prefrontal function (Mattay et al, 2003). Accordingly, in male subjects increased dopaminergic availability (within normal levels (Bertolino et al, 2003)), as associated with the Met allele, leads to enhanced functional efficiency; but, by contrast, in girls Met compromises efficiency by hyper-optimally elevating dopamine. Given that estrogen downregulates COMT function (and therefore prefrontal dopamine availability is higher in females), females are suggested to be inherently further right on this curve. However, although elevated activation is often interpreted in terms of reduced efficiency in this context (Nixon et al, 2011), the validity of such claims rests on behavioral substantiation. In the SST, increased activation has been found to inversely relate to SSRT and is, therefore, recognized as a marker of effective function (Aron et al, 2003; Aron and
Poldrack, 2006; Chambers et al, 2006). In this work, despite neurophysiological effects, neither sex, COMT nor the interaction of these factors significantly predicted SSRT, which serves as further support for the notion that COMT effects are more evident at the brain than the behavioral level (Wardle et al, 2013). Given that no significant behavioral differences were observed between sex-genotype groups, compromised efficiency in male Val carriers can be reasonably interpreted in both the mechanisms responsible for the generation and implementation of behavioral inhibition, as elevated activation does not afford any behavioral advantage. Contrary to the hypothesis that Val provides an advantage in tasks requiring cognitive flexibility (Bilder et al, 2004; Grace, 1991; Seamans and Yang, 2004)), our findings suggest that in males the Val allele is associated with inefficient cortical function during a task requiring rapid cognitive updating.

This is not the first fMRI study of COMT-gene effects on inhibition. At odds with the current observations, Congdon et al (2009) reported that Met homozygotes displayed increased activation in regions including SMA, IFC, and subthalamic nucleus. However, after categorizing for genotype, the group sizes of this study were small (Met-Met: $n=6$; Val-Met: $n=27$; Val-Val: $n=10$ ) and not split by sex, both of which limit the findings. By contrast, the large sample size of the current study greatly enhances its power to detect the effects of genetic variation on brain physiology, especially when they are small. Null 
COMT effects on right-prefrontal BOLD activation during successful inhibition have been previously reported in an overlapping sample of the IMAGEN cohort (Whelan et al, 2012). However, the factor-analysis procedure employed previously, while elegantly effective as a data-reduction technique, has potential to limit sensitivity to regionally specific effects; and the prior study did not directly assess sex moderation of COMT effects.

The pediatric focus of the current study also serves to demonstrate that the sexually dimorphic effects observed are not limited to the mature brain. A pertinent aim for future work will be to examine the mutability of the current effects across the lifecycle. It has been recently reported that activation of areas including right IFC during SST-induced inhibition is greater in early adulthood than in adolescence; and that maturation effects here are greater in males compared with females (Rubia et al, 2013). These findings and those of age-related improvement in inhibitory performance (Rubia et al, 2013) imply that mechanistic adaptation fundamentally shapes inhibitory performance; however, the changing role of genetic and hormonal factors (and related sexual dimorphism) remains unclear.

Interestingly, in light of prior null findings on sex differences in inhibition in adults (Li et al, 2006, 2009) and despite the performance-tracking algorithm, sex significantly affected both inhibitory and executive aspects of performance. Girls exhibited increased go-success and stop-success rates compared with boys, and boys produced significantly more STE responses than girls, suggestive of a more impulsive performance style in boys. It is possible that the previous studies were underpowered to detect these effects or alternatively that these effects diminish in adulthood, given evidence for superior inhibitory performance in girls relative to boys in pediatric samples (Bezdjian et al, 2009). Another possibility is that sex moderation of behavioral inhibition is variable. Women have been shown to be less efficient than men at inhibiting prepotent responses during the follicular phase of the menstrual cycle, when estradiol and dopamine turnover rates increase (Colzato et al, 2010). This suggests a statedependent change, which could be feasibly investigated in future work on COMT brain effects. It must be seen as a limitation of the current work that hormonal concentrations were not explicitly assessed. Indeed, it is possible that increased hormonal heterogeneity in girls masks COMT effects in this group; however, the current behavioral and neuroimaging data provide little evidence of greater between-subject variation of response in adolescent females.

Our finding that prefrontal BOLD activation significantly mediates the relationship between COMT and SSRT in boys but not in girls is reliable over a set of related models (Supplementary Information 6) and suggests that sex differences are apparent not only in the extent to which COMT genotype affects physiology but also in the extent to which this physiological index determines behavior. Inconsistencies in the literature on sex differences in COMT effects at a behavioral level may be attributable to the bluntness of the behavioral tools used to date; however, our findings offer an alternative-that divergent mechanistic pathways are adopted between the sexes but that these lead to convergent behavioral outcomes.
This work is predicated on and provides support for estrogen moderation of COMT activity. However, several lines of evidence suggest this model is simplistic (Tunbridge and Harrison, 2010); COMT is not only downregulated by estrogens, it also metabolizes catecholamine estrogens (Creveling, 2003). Further, the finding that COMT activity is higher in men despite reduced or equivalent mRNA levels in men compared with women (Dempster et al, 2006) suggests that transcriptional regulation is not the sole driver of COMT sex effects. Moreover, the finding that COMT protein and activity rises in men aged 20-50 years (Tunbridge et al, 2007), in the face of steady estradiol levels in this time frame (Bjornerem et al, 2004), provides further support for this notion. Future direct investigation of estrogen effects on COMT-gene activity is, therefore, strongly warranted, and would be likely enhanced if supplementary sources of potential variation were considered. For instance, COMT-by-serotonin-transporter gene interactions on limbic BOLD activation during aversivestimulus processing (Smolka et al, 2007) and COMT-byDRD4 gene interactions on prefrontal BOLD during cognitive control (Heinzel et al, 2012) indicate that COMT-gene effects are determined by complex genetic associations affecting multiple, interacting neurotransmitter systems. Furthermore, future investigation of the influence of epigenetic and environmental factors on the reported COMT effects is suggested, in view of the differential cytosine-p-guanine site characteristics of the $\mathrm{Val}$ and Met alleles, potential modulation of methylation by stress (Jirtle and Skinner, 2007), and recent observations that Val-allele methylation interacts with stress to modify prefrontal BOLD activation during working memory (Ursini et al, 2011). Discrepancies in the COMT literature may be partially attributable to such factors. This notion is additionally supported by recent animal work revealing a complex and discrete pattern of interactions between sex, COMT genotype and stress on inhibition-related behaviors (Papaleo et al, 2012).

Focus on alternative neuroendocrine systems can also potentially offer an improved perspective on the currently observed phenomena. Specifically, it can be reasonably hypothesized that testosterone directly amplifies prefrontal COMT effects in adolescent males, in light of sex differences in its circulating levels (Granger et al, 1999), and recent observations that it modulates dopaminergic tone in the rat substantia nigra via regulation of enzymes implicated in dopamine metabolism and transportation (Purves-Tyson et al, 2012). It is nevertheless noted that testosterone effects on COMT have, to date, been inconsistently observed (Purves-Tyson et al, 2014).

By demonstrating sexual dimorphism in COMT effects on cerebral activation in the healthy brain, this work extends our understanding of the mechanisms underpinning cognition in prefrontal cortex. Given that sex shapes genotypic expression (and phenotypic sequelae), by virtue of differential hormonal and epigenetic factors, it is seemingly vital to account for its effects when studying genetic control of cognition. This has important implications not only for our understanding of the genetic determinants of normal cognition but also for the factors which lead to contrasting patterns of psychopathology in males and females. 


\section{FUNDING AND DISCLOSURE}

GJB has received honoraria for teaching from GE Healthcare, and acts as a consultant for IXICO. The remaining authors declare no conflicts of interest.

\section{ACKNOWLEDGEMENTS}

This work was supported by the European Union-funded FP6 Integrated Project IMAGEN (Reinforcement-related behaviour in normal brain function and psychopathology) (LSHM-CT2007-037286), the FP7 projects ADAMS (Genomic variations underlying common neuropsychiatric diseases and disease related cognitive traits in different human populations) (242257) and the Innovative Medicine Initiative Project EUAIMS (115300-2), as well as the United Kingdom National Institute for Health Research Biomedical Research Centre Mental Health and the Medical Research Council Programme Grant 'Developmental pathways into adolescent substance abuse' (93558), the Swedish Research Council (FORMAS) and the Deutsche Forschungsgemeinschaft (DFG Grant SFB 940/1).

\section{REFERENCES}

Aron AR (2011). From reactive to proactive and selective control: developing a richer model for stopping inappropriate responses. Biol Psychiatry 69: e55-e68.

Aron AR, Fletcher PC, Bullmore ET, Sahakian BJ, Robbins TW (2003). Stop-signal inhibition disrupted by damage to right inferior frontal gyrus in humans. Nat Neurosci 6: 115-116.

Aron AR, Poldrack RA (2006). Cortical and subcortical contributions to Stop signal response inhibition: role of the subthalamic nucleus. J Neurosci 26: 2424-2433.

Barnett JH, Heron J, Goldman D, Jones PB, Xu K (2009). Effects of catechol-O-methyltransferase on normal variation in the cognitive function of children. Am J Psychiatry 166: 909-916.

Barnett JH, Heron J, Ring SM, Golding J, Goldman D, Xu K et al (2007). Gender-specific effects of the catechol-O-methyltransferase Val108/158Met polymorphism on cognitive function in children. Am J Psychiatry 164: 142-149.

Becker A, Steinhausen HC, Baldursson G, Dalsgaard S, Lorenzo MJ, Ralston SJ et al (2006). Psychopathological screening of children with ADHD: Strengths and Difficulties Questionnaire in a panEuropean study. Eur Child Adolesc Psychiatry 15(Suppl 1): I56-I62.

Bertolino A, Frye M, Callicott JH, Mattay VS, Rakow R, SheltonRepella J et al (2003). Neuronal pathology in the hippocampal area of patients with bipolar disorder: a study with proton magnetic resonance spectroscopic imaging. Biol Psychiatry 53: 906-913.

Bezdjian S, Baker LA, Lozano DI, Raine A (2009). Assessing inattention and impulsivity in children during the Go/NoGo task. Br J Dev Psychol 27(Pt 2): 365-383.

Bilder RM, Volavka J, Lachman HM, Grace AA (2004). The catechol-O-methyltransferase polymorphism: relations to the tonic-phasic dopamine hypothesis and neuropsychiatric phenotypes. Neuropsychopharmacology 29: 1943-1961.

Bjornerem A, Straume B, Midtby M, Fonnebo V, Sundsfjord J, Svartberg J et al (2004). Endogenous sex hormones in relation to age, sex, lifestyle factors, and chronic diseases in a general population: the Tromso Study. J Clin Endocrinol Metab 89: 6039-6047.

Campbell A, Muncer S (2009). Can 'risky' impulsivity explain sex differences in aggression? Pers Individ Differ 47: 402-406.

Carskadon MA, Acebo C (1993). A self-administered rating scale for pubertal development. J Adolesc Health 14: 190-195.
Chambers CD, Bellgrove MA, Stokes MG, Henderson TR, Garavan H, Robertson IH et al (2006). Executive 'brake failure' following deactivation of human frontal lobe. J Cogn Neurosci 18: 444-455.

Chambers CD, Garavan H, Bellgrove MA (2009). Insights into the neural basis of response inhibition from cognitive and clinical neuroscience. Neurosci Biobehav Rev 33: 631-646.

Chen J, Lipska BK, Halim N, Ma QD, Matsumoto M, Melhem S et al (2004). Functional analysis of genetic variation in catecholO-methyltransferase (COMT): effects on mRNA, protein, and enzyme activity in postmortem human brain. Am J Hum Genet 75: 807-821.

Cohn CK, Axelrod J (1971). The effect of estradiol on catechol-Omethyltransferase activity in rat liver. Life Sci I 10: 1351-1354.

Colzato LS, Hertsig G, van den Wildenberg WP, Hommel B (2010). Estrogen modulates inhibitory control in healthy human females: evidence from the stop-signal paradigm. Neuroscience 167: 709-715.

Coman IL, Gnirke MH, Middleton FA, Antshel KM, Fremont W, Higgins AM et al (2010). The effects of gender and catechol O-methyltransferase (COMT) Val108/158Met polymorphism on emotion regulation in velo-cardio-facial syndrome (22q11.2 deletion syndrome): an fMRI study. Neuroimage 53: 1043-1050.

Congdon E, Constable RT, Lesch KP, Canli T (2009). Influence of SLC6A3 and COMT variation on neural activation during response inhibition. Biol Psychol 81: 144-152.

Creveling CR (2003). The role of catechol-O-methyltransferase in the inactivation of catecholestrogen. Cell Mol Neurobiol 23: 289-291.

Dale AM (1999). Optimal experimental design for event-related fMRI. Hum Brain Mapp 8: 109-114.

Dempster EL, Mill J, Craig IW, Collier DA (2006). The quantification of COMT mRNA in post mortem cerebellum tissue: diagnosis, genotype, methylation and expression. BMC medical genetics 7: 10.

Domschke K, Baune BT, Havlik L, Stuhrmann A, Suslow T, Kugel H et al (2012). Catechol-O-methyltransferase gene variation: impact on amygdala response to aversive stimuli. Neuroimage 60: 2222-2229.

Egan MF, Goldberg TE, Kolachana BS, Callicott JH, Mazzanti CM, Straub RE et al (2001). Effect of COMT Val108/158 Met genotype on frontal lobe function and risk for schizophrenia. Proc Natl Acad Sci USA 98: 6917-6922.

Eme RF (2007). Sex differences in child-onset, life-coursepersistent conduct disorder. A review of biological influences. Clin Psychol Rev 27: 607-627.

Grace AA (1991). Phasic versus tonic dopamine release and the modulation of dopamine system responsivity: a hypothesis for the etiology of schizophrenia. Neuroscience 41: 1-24.

Granger DA, Schwartz EB, Booth A, Arentz M (1999). Salivary testosterone determination in studies of child health and development. Horm Behav 35: 18-27.

Harrison PJ, Tunbridge EM (2008). Catechol-O-methyltransferase (COMT): a gene contributing to sex differences in brain function, and to sexual dimorphism in the predisposition to psychiatric disorders. Neuropsychopharmacology 33: 3037-3045.

Hart H, Radua J, Nakao T, Mataix-Cols D, Rubia K (2013). Metaanalysis of functional magnetic resonance imaging studies of inhibition and attention in attention-deficit/hyperactivity disorder: exploring task-specific, stimulant medication, and age effects. JAMA Psychiatry 70: 185-198.

Hayes AF (2013). Introduction to Mediation, Moderation, and Conditional Process Analysis: A Regression-Based Approach (Methodology in the Social Sciences). Guilford Press: New York.

Heinzel S, Dresler T, Baehne CG, Heine M, Boreatti-Hummer A, Jacob CP et al (2012). COMT x DRD4 epistasis impacts prefrontal cortex function underlying response control. Cereb Cortex 23: 1453-1462. 
Jirtle RL, Skinner MK (2007). Environmental epigenomics and disease susceptibility. Nat Rev Genet 8: 253-262.

Kates WR, Antshel KM, Abdulsabur N, Colgan D, Funke B, Fremont $\mathrm{W}$ et al (2006). A gender-moderated effect of a functional COMT polymorphism on prefrontal brain morphology and function in velo-cardio-facial syndrome (22q11.2 deletion syndrome). Am J Med Genet B, Neuropsychiatr Genet 141B: 274-280.

Kessler RC, Angermeyer M, Anthony JC, DE Graaf R, Demyttenaere K, Gasquet I et al (2007). Lifetime prevalence and age-of-onset distributions of mental disorders in the World Health Organization's World Mental Health Survey Initiative. World Psychiatry 6: 168-176.

Labouvie EW, McGee CR (1986). Relation of personality to alcohol and drug use in adolescence. J Consult Clin Psychol 54: 289-293.

Li CS, Huang C, Constable RT, Sinha R (2006). Gender differences in the neural correlates of response inhibition during a stop signal task. Neuroimage 32: 1918-1929.

Li CS, Zhang S, Duann JR, Yan P, Sinha R, Mazure CM (2009). Gender Differences in Cognitive Control: an Extended Investigation of the Stop Signal Task. Brain Imaging Behav 3: 262-276.

Logan GD, Cowan WB, Davis KA (1984). On the ability to inhibit simple and choice reaction time responses: a model and a method. J Exp Psychol Hum Percept Perform 10: 276-291.

Maldjian JA, Laurienti PJ, Kraft RA, Burdette JH (2003). An automated method for neuroanatomic and cytoarchitectonic atlas-based interrogation of fMRI data sets. Neuroimage 19: 1233-1239.

Mattay VS, Goldberg TE, Fera F, Hariri AR, Tessitore A, Egan MF et al (2003). Catechol O-methyltransferase val158-met genotype and individual variation in the brain response to amphetamine. Pro Natl Acad Sci USA 100: 6186-6191.

Mier D, Kirsch P, Meyer-Lindenberg A (2010). Neural substrates of pleiotropic action of genetic variation in COMT: a meta-analysis. Mol Psychiatry 15: 918-927.

Newman JP, MacCoon DG, Vaughn LJ, Sadeh N (2005). Validating a distinction between primary and secondary psychopathy with measures of Gray's BIS and BAS constructs. J Abnorm Psychol 114: 319-323.

Nixon DC, Prust MJ, Sambataro F, Tan HY, Mattay VS, Weinberger DR et al (2011). Interactive effects of DAOA (G72) and catechol-O-methyltransferase on neurophysiology in prefrontal cortex. Biol Psychiatry 69: 1006-1008.

Papaleo F, Erickson L, Liu G, Chen J, Weinberger DR (2012). Effects of sex and COMT genotype on environmentally modulated cognitive control in mice. Pro Natl Acad Sci USA 109: 20160-20165.

Purves-Tyson TD, Handelsman DJ, Double KL, Owens SJ, Bustamante S, Weickert CS (2012). Testosterone regulation of sex steroid-related mRNAs and dopamine-related mRNAs in adolescent male rat substantia nigra. BMC Neurosci 13: 95.

Purves-Tyson TD, Owens SJ, Double KL, Desai R, Handelsman DJ, Weickert CS (2014). Testosterone induces molecular changes in dopamine signaling pathway molecules in the adolescent male rat nigrostriatal pathway. PLoS One 9: e91151.

Rosa EC, Dickinson D, Apud J, Weinberger DR, Elvevag B (2010). COMT Val158Met polymorphism, cognitive stability and cognitive flexibility: an experimental examination. Behav Brain Funct 6: 53.

Rubia K, Halari R, Cubillo A, Smith AB, Mohammad AM, Brammer $M$ et al (2011). Methylphenidate normalizes frontostriatal underactivation during interference inhibition in medication-naive boys with attention-deficit hyperactivity disorder. Neuropsychopharmacology 36: 1575-1586.

Rubia K, Lim L, Ecker C, Halari R, Giampietro V, Simmons A et al (2013). Effects of age and gender on neural networks of motor response inhibition: From adolescence to mid-adulthood. Neuroimage 83C: 690-703.

Rubia K, Noorloos J, Smith A, Gunning B, Sergeant J (2003). Motor timing deficits in community and clinical boys with hyperactive behavior: the effect of methylphenidate on motor timing. J Abnorm Child Psychol 31: 301-313.

Rubia K, Smith AB, Taylor E, Brammer M (2007). Linear agecorrelated functional development of right inferior frontostriato-cerebellar networks during response inhibition and anterior cingulate during error-related processes. Hum Brain Mapp 28: 1163-1177.

Schumann G, Loth E, Banaschewski T, Barbot A, Barker G, Buchel C et al (2010). The IMAGEN study: reinforcement-related behaviour in normal brain function and psychopathology. Mol Psychiatry 15: 1128-1139.

Seamans JK, Yang CR (2004). The principal features and mechanisms of dopamine modulation in the prefrontal cortex. Prog Neurobioly 74: 1-58.

Smolka MN, Buhler M, Schumann G, Klein S, Hu XZ, Moayer M et al (2007). Gene-gene effects on central processing of aversive stimuli. Mol Psychiatry 12: 307-317.

Tahmasebi AM, Artiges E, Banaschewski T, Barker GJ, Bruehl R, Buchel C et al (2012). Creating probabilistic maps of the face network in the adolescent brain: a multicentre functional MRI study. Hum Brain Mapp 33: 938-957.

Tanner JM (1962). Growth at adolescence. Blackwell: Oxford.

Tunbridge EM, Harrison PJ (2010). Importance of the COMT Gene for Sex Differences in Brain Function and Predisposition to Psychiatric Disorders. In: Neill JC, Kulkarni J (eds) Biological Basis of Sex Differences in Psychopharmacology. Springer-Verlag: Berlin. Tunbridge EM, Weickert CS, Kleinman JE, Herman MM, Chen J, Kolachana BS et al (2007). Catechol-o-methyltransferase enzyme activity and protein expression in human prefrontal cortex across the postnatal lifespan. Cereb Cortex 17: 1206-1212.

Tzourio-Mazoyer N, Landeau B, Papathanassiou D, Crivello F, Etard O, Delcroix N et al (2002). Automated anatomical labeling of activations in SPM using a macroscopic anatomical parcellation of the MNI MRI single-subject brain. Neuroimage 15: 273-289.

Ursini G, Bollati V, Fazio L, Porcelli A, Iacovelli L, Catalani A et al (2011). Stress-related methylation of the catechol-O-methyltransferase Val 158 allele predicts human prefrontal cognition and activity. J Neurosci 31: 6692-6698.

Wardle MC, de Wit H, Penton-Voak I, Lewis G, Munafo MR (2013). Lack of association between COMT and working memory in a population-based cohort of healthy young adults. Neuropsychopharmacology 38: 1253-1263.

Wechsler D, Golombok S, Rust J (1992). Wechsler Intelligence Scale for Children. 3rd. edn Tex, Psychological Corp.: San Antonio.

Whelan R, Conrod PJ, Poline JB, Lourdusamy A, Banaschewski T, Barker GJ et al (2012). Adolescent impulsivity phenotypes characterized by distinct brain networks. Nat Neurosci 15: 920-925.

Xie T, Ho SL, Ramsden D (1999). Characterization and implications of estrogenic down-regulation of human catechol-O-methyltransferase gene transcription. Mol Pharmacol 56: 31-38.

Yavich L, Forsberg MM, Karayiorgou M, Gogos JA, Mannisto PT (2007). Site-specific role of catechol-O-methyltransferase in dopamine overflow within prefrontal cortex and dorsal striatum. J Neurosci 27: 10196-10209.

Zinkstok J, Schmitz N, van Amelsvoort T, de Win M, van den Brink W, Baas F et al (2006). The COMT val158met polymorphism and brain morphometry in healthy young adults. Neurosci Lett 405: 34-39.

Supplementary Information accompanies the paper on the Neuropsychopharmacology website (http://www.nature.com/npp) 